\title{
Actualización en el diagnóstico y tratamiento de la Fascitis plantar.
}

DOI: http//dx.doi.org/10.37315/SOTOCAV202028455140

MIÑANO-MARTÍNEZ I.

SERVICIO DE MEDICINA FÍSICA Y REHABILITACIÓN HOSPITAL CLÍNICO UNIVERSITARIO DE VALENCIA.

\section{Resumen.}

La fascitis plantar es una patología muy frecuente. A lo largo del tiempo, algunos aspectos de esta entidad han generado controversia, por lo que nuestro objetivo es agrupar la última evidencia científica disponible. La fascitis plantar tiene su origen en un proceso degenerativo consecuencia de microtraumatismos de repetición. Además, intervienen múltiples factores de riesgo que contribuyen a la sobrecarga de la fascia y a su degeneración colágena. Su diagnóstico es clínico, reservándose la realización de pruebas complementarias para realizar diagnóstico diferencial con otras entidades, que pueden tener origen en la fascia plantar o en estructuras adyacentes, debiendo prestar especial atención a los atrapamientos nerviosos. Existen numerosos tratamientos que han demostrado efectividad, obteniendo mejores resultados individualizando a cada paciente y con la combinación de tratamientos.

Palabras clave: plantar fasciitis, plantar fasciopathy, talar heel pain.

\section{Summary.}

Plantar fasciitis is a very common pathology. Over time, some aspects of this entity have generated controversy, so our objective is to group the latest available scientific evidence. Plantar fasciitis has its origin in a degenerative process resulting from repetitive microtrauma. In addition, multiple risk factors are involved that contribute to its overload the fascia and its collagen degeneration. Its diagnosis is clinical, reserving the performance of complementary tests to perform differential diagnosis with other entities, which may originate in the plantar fascia or adjacent structures, paying special attention to nerve entrapments. There are numerous treatments that have proven effectiveness, obtaining better results by individualizing each patient and with a combination of treatments.

\section{Correspondencia:}

Isabel Miñano Martínez.

isabelmimar@gmail.com 


\section{Introducción}

La fascitis plantar es una patología muy frecuente. Su prevalencia en la población general es del $3.6 \%$ al $7 \%{ }^{1}$, suponiendo el $8 \%$ de todas las lesiones en deportistas ${ }^{2}$. Hasta el $15 \%$ consultas de Atención Primaria ${ }^{3}$.

Es importante realizar un breve repaso de la anatomía de la planta del pie y recordar que la fascia plantar es una banda de tejido conectivo formada por fibras de colágeno, hiluronidasa y corpúsculos de Paccini y Ruffini. Se origina en el calcáneo y tiene 3 segmentos: el más importante es el segmento central, que se dirige a las falanges proximales; el segmento lateral, que se dirige al $5^{\circ}$ metatarsiano, y el segmento medial, que cubre al abductor del $1^{\circ}$ dedo. Además, se relaciona con el tríceps sural mediante el tendón de Aquiles ${ }^{4,5}$. De esta forma podemos encontrarnos con una fascitis plantar insercional, no insercional o con la afectación de cualquiera de estas bandas ${ }^{1}$.

Bajo la fascia plantar encontramos el músculo flexor corto de los dedos, el abductor del quinto dedo y el abductor del hallux. Más en profundidad se encuentran el cuadrado plantar y el flexor largo de los dedos ${ }^{5}$.

La función de la fascia plantar es soportar el arco longitudinal plantar durante la estática y durante la dinámica de la marcha se elonga y contrae mediante el mecanismo de Windlass ${ }^{4}$.

Aspectos importantes acerca de la fascitis plantar tales como su etiología, sus factores de riesgo, su diagnóstico o su tratamiento han sido muy discutidos a lo largo del tiempo. El principal objetivo de este estudio es agrupar la última evidencia científica disponible acerca de esta entidad.

\section{Material y método}

Diseño

Se realizó una extensa investigación entre la literatura internacional para la selección de artículos mediante el motor de búsqueda PubMed, que corresponde a la base de datos de Medline, ofrecida por la Biblioteca Nacional de Medicina de los Estados Unidos.

\section{Estrategia de búsqueda}

En primer lugar, para la búsqueda utilizamos las siguientes palabras clave por separado y en combinación: plantar fasciitis, plantar fasciopathy, talar heel pain. Para la búsqueda de términos, fue utilizado el vocabulario estructurado trilingüe DeCS (descriptores en ciencias de la salud) desarrollado a partir del MeSH (medical Subject Headings de la U.S National Libary of Medine) con el objetivo de permitir el uso de terminología común para la búsqueda en tres idiomas. Los conceptos que lo componen se encuentran organizados en una estructura jerárquica, permitiendo una búsqueda en términos más amplios o específicos, o todos los términos que pertenezcan a una misma estructura jerárquica.

Además, se aplicaron filtros para acotar los resultados a ensayos clínicos y revisiones sistemáticas publicadas en los últimos 10 años. Se tomaron en consideración aquellos artículos escritos en inglés y en español. Para la obtención de los artículos más actuales se aplicó el filtro "published in the last 5 years".

Se analizaron a su vez las referencias bibliográficas de los artículos seleccionados con la finalidad de rescatar otros estudios potencialmente incluibles para la revisión.

Por último, se revisaron diversos artículos y libros relacionados directamente con la patología objeto del estudio, a los que se había llegado a partir de los artículos de interés o bien a través de consulta en archivo.

\section{Criterios de inclusión y exclusión}

Para llevar a cabo la selección de artículos, se emplearon los siguientes criterios de inclusión: Se escogieron artículos desde los últimos 10 años, para llevar a cabo una comparación de los tratamientos y técnicas diagnósticas a lo largo del tiempo. En la primera selección de artículos, se tuvo en cuanta aquellos que estuvieran directamente relacionados con la fasciopatía o fascitis plantar desde el punto de vista médico, seleccionando aquellos que aportaban más información y evitando la repetición de contenido. En el momento de preseleccionar artículos, el factor de impacto de la revista no fue tomado en cuenta. Se limitó la búsqueda a aquellos artículos redactados en inglés o español. Se excluyeron aquellos trabajos en los que la casuística era escasa o presentación de casos clínicos aislados.

\section{Etiología y factores de riesgo}

La etiología de la fascitis plantar no es del todo bien conocida. Últimos estudios han concluido que se trata de un proceso degenerativo en el que se produce un aumento de la sustancia mucoide, degeneración colágena, hiperplasia fibrógena y calcificación ${ }^{6}$, consecuencia de traumatismos de repetición que producen microrroturas en la fascia que provocan degeneración de colágeno. Como consecuencia de los microtraumatismos repetidos puede producirse una reacción inflamatoria, pero no es un proceso inflamatorio primario ${ }^{7}$.

Se considera multifactorial con diversos factores de riesgo implicados en su desarrollo ${ }^{1}$ (Fig. 1-3). La combinación de distintos factores de riesgo puede generar una sobrecarga patológica de la fascia plantar dando lugar a microtraumatismos de repetición ${ }^{7}$. 
FACTORES DE RIESGO MECÁNICOS INTRINNSECOS

Mayor edad.

IMC elevado.

Limitación de la dorsiflexión del tobillo.

Acortamiento tendón de Aquiles, tríceps sural e

isquiotibiales.

Espolón calcáneo.

Aumento del grosor de la fascia plantar.

Disminución de la almohadilla grasa talar.

Niveles bajos de estrógenos en mujeres atletas.

Disminución de la vascularización ligamentosa.

Género.

Genética.

Figura 1. Factores de riesgo mecánicos intrínsecos.

FACTORES DE RIESGO MECÁNICOS EXTRINNSECOS

Deporte: corredores.

Calzado inadecuado.

Trabajo en bipedestación prolongada con carga de

peso.

Aumento o disminución excesiva del arco plantar,

pronación del pie.

Hállux valgus.

Incrementos rápidos de actividad.

Figura 2. Factores de riesgo mecánicos extrínsecos.

\section{FACTORES DE RIESGO NO MECÁNICOS}

Diabetes.

Artritis reumatoide.

Espondilitis anquilosante.

Quimioterapia.

Infecciones retrovirales.

Figura 3. Factores de riesgo no mecánicos.

Entre los factores de riesgo encontramos la edad avanzada, la obesidad, la disminución de la almohadilla grasa y un nivel de estrógenos bajo en mujeres atletas $^{1,3}$. Estos factores tienen en común los microtraumatismos de repetición y la degeneración colágena de la fascia plantar, lo que está en consonancia con los cambios degenerativos encontrados en estudios histológicos ${ }^{8}$ y apoya el origen de que la fascitis plantar es secundaria a microtraumatismos $^{1,7}$. El aumento del grosor de la fascia plantar, que se traduce en una disminución de la elasticidad; la presencia de hallux valgus ${ }^{9}$ y la pronación excesiva del pie disminuyen la capacidad de absorber impactos y resistir las fuerzas de tensión normales ${ }^{1}$.

La alteración del mecanismo de Windlass ha sido ampliamente relacionado con la fascitis plantar en la literatura $^{1,10}$. Entre los factores que contribuyen a su alteración encontramos el acortamiento de la musculatura de la cadena posterior y, tendón de Aquiles y tríceps sural ${ }^{11}$, así como la limitación de la dorsiflexión del tobillo $1,3,10$. Este último es considerado el factor más importante para algunos estudios aunque no está claro que sea causa o consecuencia de la fascitis plantar $^{1}$.

En cuanto al género, no encontramos resultados consistentes en la literatura, aunque se encuentra peor pronóstico de la fascitis plantar en mujeres y cuando es bilateral ${ }^{11}$. Por otro lado, se han relacionado algunas variantes genéticas con el desarrollo de fascitis plantar ${ }^{1}$.

Esta entidad está asociada con la carga de trabajo y el deporte, encontramos una prevalencia aumentada en corredores asociada a lesión por sobreuso en carrera, técnica pobre, errores en el entrenamiento y elevada intensidad del mismo, sobrecarga repetida, fatiga, disfunción y poca flexibilidad de la musculatura de los miembros inferiores, y uso de calzado inadecuado ${ }^{1}$. También encontramos mayor incidencia en aquellos trabajos en bipedestación prolongada sobretodo si se realizan sobre superficies dura y con carga de peso ${ }^{3,13}$.

Se relacionan estrechamente con la fascitis plantar por afectar a la micro y macrovascularización, el tratamiento con quimioterápicos y la diabetes mellitus ${ }^{14}$. Además, de las enfermedades inflamatorias sistémicas tales como la artritis reumatoide, espondilitis anquilopoyética, enfermedad de Reiter o gota ${ }^{1}$.

\section{Diagnóstico}

El diagnóstico de fascitis plantar es clínico, basado en la anamnesis y la exploración física. En principio no son necesarias pruebas complementarias; no obstante las pruebas de imagen o de laboratorio ayudan a realizar un diagnóstico diferencial con otras patologías ${ }^{3,15}$.

La presentación típica es un dolor agudo en zona anterointerna del talón, muy intenso con los primeros pasos de la mañana, a continuación va disminuyendo y vuelve a aumentar al final del día, sobretodo en relación con la bipedestación prolongada o actividades de impacto.

En la exploración física objetivamos dolor a la palpación de tuberosidad medial del calcáneo o en la inserción proximal de la fascia plantar, que puede aumentar con la extensión de los dedos. Puede asociarse una limitación de la flexión dorsal de tobillo, alteración del test de Windlass y negatividad del tinnel tarsiano ${ }^{3}$.

Podemos apoyarnos en el test Jack para valorar alteración del mecanismo de windlass o en el test de Silverskiold en caso de encontrar limitación de la flexión dorsal del tobillo para valorar acortamiento de la musculatura del tríceps sural.

En cuanto a pruebas complementarias en las que podemos apoyar el diagnóstico de fascitis plantar encontramos las pruebas de imagen ${ }^{16}$, las pruebas de laboratorio y los estudios neurofisiológicos (Fig. 4). Hay 
estudios que indican que las pruebas complementarias deben reservarse a casos de fuerte sospecha de otro diagnóstico: pacientes que no mejoran lo esperado después del tratamiento inicial o que presentan una historia clínica o una exploración física anómalas. No obstante, otros recomiendan realizar pruebas de imagen para asegurarse de que no se trata de otra causa de talalgia, como un tumor ${ }^{17}$.

\begin{tabular}{|l|l|}
\hline PRUEBAS DE IMAGEN & OTRAS \\
\hline Radiografia simple. & $\begin{array}{l}\text { Analitica sanguínea con marcadores } \\
\text { inflamatorios. }\end{array}$ \\
\hline RM. & EMG. \\
\hline Ecografia. & Estudio biomecánico de la marcha. \\
\hline
\end{tabular}

Figura 4. Pruebas diagnósticas complementarias.

En una radiografía simple apreciamos signos inflamatorios, fracturas, la presencia de espolón calcáneo, que si bien puede aparecer en asociación con fascitis plantar, su sola presencia es insuficiente para considerarlo la causa ${ }^{6,15,16}$. Realizadas en carga permiten descartar fallos arquitectónicos que afecten a la biomecánica ${ }^{10}$.

En algunas ocasiones necesitamos hacer uso de la resonancia magnética (RM) para el diagnóstico de fascitis plantar. En ella encontraremos engrosamiento de la fascia con hiperintensidad de señal en T2 (normalmente presenta densidad homogénea y baja en todas las secuencias), tejido subcutáneo adyacente e inserción calcánea (Fig. 5). También podemos ver fractura de estrés de calcáneo y edema óseo ${ }^{15,17}$.

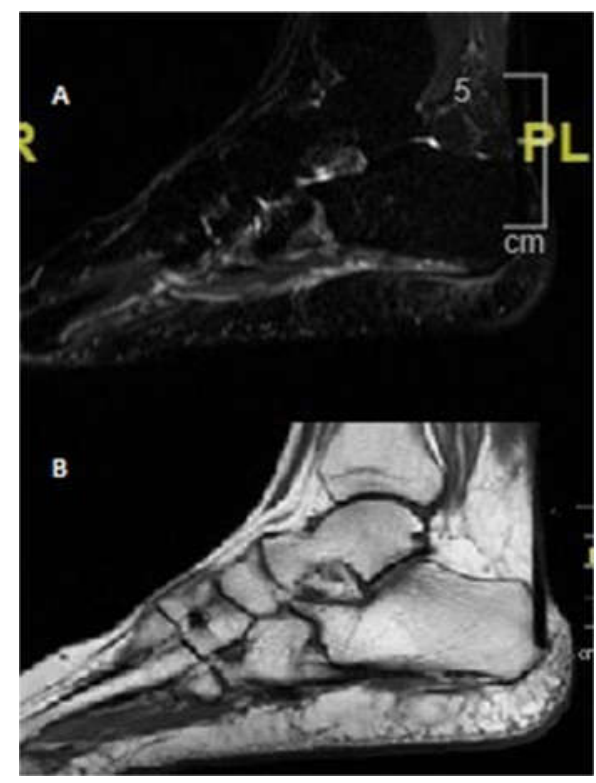

Figura 5: Corte sagital de RNM T2(A) y T1(B) donde se observa engrosamiento y cambios inflamatorios a nivel de inserción el calcáneo de la fascia plantar.

La ecografía ha demostrado tener un gran valor para el diagnóstico de fascitis plantar ${ }^{1,3,18}$ (Fig. 6). El valor de corte del grosor de la fascia plantar es de más $4 \mathrm{~mm}$ para fascitis plantares insercionales (sensibilidad: $98,8 \%$ y especifidad: $88,8 \%$ ) que mejora cuando el corte es en $4,41 \mathrm{~mm}^{18}$, demostrando buena reproducibilidad intra e interobservador ${ }^{19}$ y una buena correlación con las medidas de $\mathrm{RM}^{20}$.

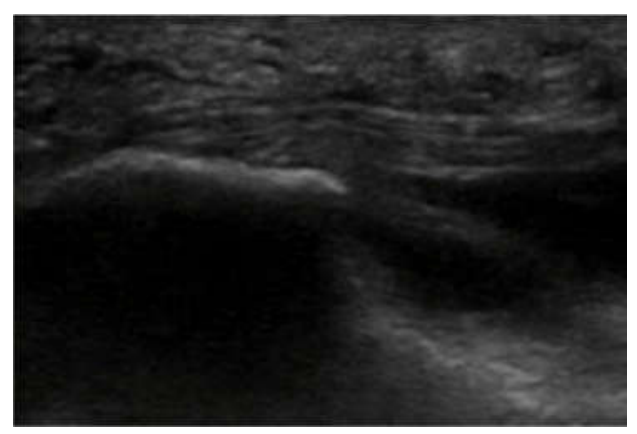

Figura 6. Imagen ecográfica de fascitis plantar.

Otros estudios nos pueden ayudar a realizar el diagnóstico diferencial y detectar alteraciones. La analítica sanguínea, que resulta útil para estudiar marcadores inflamatorios ante sospecha de enfermedades inflamatorias tales como artritis reumatoide o la enfermedad de Reiter o incluso metabólicas como la gota ${ }^{21}$.

El estudio biomecánico de la marcha permite estudiar alteraciones biomecánicas. La electromiografía para estudiar radiculopatías o posibles atrapamientos nerviosos rara vez es necesaria y puede ser normal en casos de atrapamiento nervioso.

\section{Diagnóstico diferencial}

Hay numerosas patologías que son causa de talalgia, siendo la más común la fascitis plantar. Según la localización exacta y el tipo de dolor, sobre todo cuando no nos cuadra con el cuadro típico de fascitis plantar, debemos tener en cuenta otras entidades (Fig. 7).

El espolón calcáneo se forma como consecuencia de estrés mecánico y/o inflamatorio ${ }^{3}$. Su presencia se ha asociado tradicionalmente con la sintomatología de la fascitis plantar, aunque a día de hoy la literatura ha concluido que no necesariamente hay correlación ${ }^{10}$, los pacientes con espolón tienen 4,6 veces más probabilidades de tener $o$ haber tenido dolor que pacientes que no lo presentan ${ }^{3}$.

Por otro lado, un gran porcentaje (10-65\%) de los pacientes con espolón no presentan dolor y otros, tras la escisión del mismo, continúan con síntomas. Esto último puede ser debido a la persistencia de la sobrecarga mecánica, inflamación asociada, atrofia grasa de la almohadilla plantar, fracturas del espolón y compresión nerviosa ${ }^{6}$. 


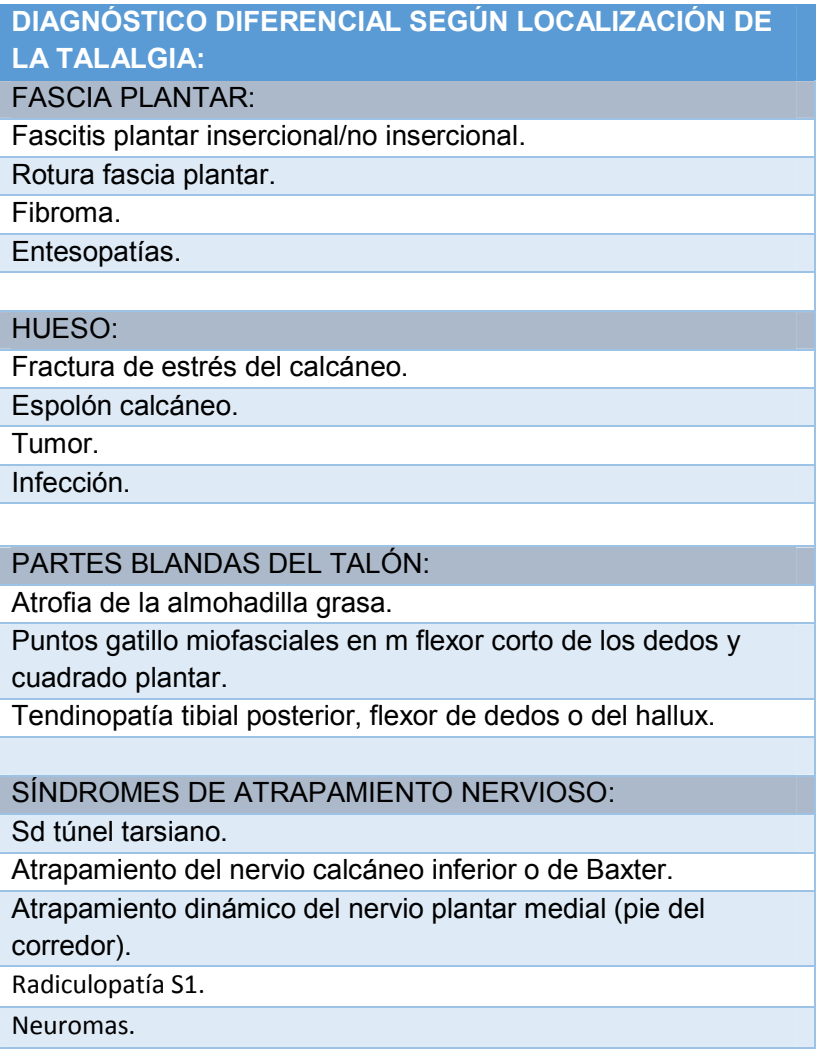

Figura 7. Diagnóstico diferencial según localización de la talalgia.

El síndrome de la almohadilla grasa es resultado de la degeneración de la grasa talar. Ésta está formada por tabiques colágenos y se encarga de las propiedades mecánicas de la almohadilla del talón. En su degeneración intervienen factores de riesgo tales como la edad aumentada, factores mecánicos, metabólicos o iatrogénicos (como la infiltración con corticoides) ${ }^{22}$.

La lesión nerviosa puede ser causa de dolor o acompañar a una fascitis plantar ${ }^{23}$. Es conveniente la realización de una anamnesis y exploración físicas detalladas para detectar características de dolor neuropático tales como tinnel positivo en cara interna del talón o en región insercional en pacientes con espolón voluminoso, o la presencia de parestesias o disestesias. El estudio electromiográfico suele ser negativo y podemos ver atrofia en RM en gente asintomática en los músculos inervados por la rama atrapada.

Los nervios que se pueden lesionar son el nervio tibial y sus ramas. Pueden sufrir atrapamiento nervioso o la formación de neuromas ${ }^{15,24}$. La primera es el nervio calcáneo medial, que va a inervar el talón y la da antes de entrar en el túnel del tarso. Después da 2 ramas: el nervio plantar medial, que recorre la región medial de la planta del pie hacia $1^{\circ}, 2^{\circ}$ y $3^{\circ}$ dedos; y el nervio plantar lateral que irá hacia $4^{\circ}$ y $5^{\circ}$ dedos. Este nervio plantar lateral su primera rama será el nervio calcáneo inferior o de Baxter, que pasa entre el músculo cuadrado plantar y la fascia plantar hacia la región lateral ${ }^{5}$. Los síndromes de atrapamiento más importantes son el síndrome del túnel tarsiano y el atrapamiento del nervio de Baxter, siendo este último el que provoca mayor confusión con la fascitis plantar ${ }^{1,15}$. Los neuromas no siempre van a producir un dolor neuropático, también pueden producir dolor de características mecánicas y síndromes de atrapamiento de los nervios adyacentes al comprimirlos $^{23}$.

\section{Tratamiento}

En la bibliografía existen múltiples tratamientos para la fascitis plantar, que ordenamos en 3 escalones terapéuticos (Fig. 8).

\begin{tabular}{|c|}
\hline PRIMER ESCALÓN TERAPÉUTICO \\
\hline Acolchado del pie y modificaciones del calzado. \\
Antiinflamatorios orales. \\
\hline Estiramientos de fascia plantar y tríceps sural. \\
Crioterapia domiciliaria. \\
Limitación de la actividad. \\
Infiltraciones de corticoides. \\
\hline SEGUNDO ESCALÓN TERAPÉUTICO \\
Dispositivos ortopédicos. \\
Férulas nocturnas. \\
\hline Tratamiento fisioterápico. \\
\hline 2a infiltración con corticoides. \\
\hline PRP. \\
\hline Infiltración con otras sustancias tales como BTX-A, \\
\hline Pnmovilización con férula de yeso o Cam Walker. \\
\hline \\
\hline Fasciotomía plantar endoscópica o cirugía \\
mínimamente invasiva. \\
\hline Radiofrecuencia bipolar. \\
\hline \\
\hline
\end{tabular}

Figura 8. Escalones terapéuticos.

El tratamiento inicial debe ser simple y coste efectivo, incluyendo ejercicios de estiramientos de fascia plantar y tríceps sural, calzado cómodo acolchado, plantillas de soporte de arco medial y acolchado del talón, toma de antiinflamatorios vía oral ${ }^{10}$ como celecoxib $^{15}$, crioterapia domiciliaria, reposo relativo evitando deporte de impacto y bipedestación prolongada, pérdida de peso $^{25}$ e inyecciones con corticoesteroides ${ }^{3,10,25}$, que deberán usarse con cuidado por haberse relacionado con rotura de la fascia y atrofia de la almohadilla grasa ${ }^{15}$. 
Si el escalón inicial produce mejoría total o parcial, continuar la terapia hasta la resolución de los síntomas. Si por el contrario, tras 6 semanas no resulta satisfactorio, se debe pasar a un segundo escalón terapéutico que incluye el uso de férulas nocturnas para mantener la extensión de la fascia plantar y el complejo gastrosóleo durante el sueño, prescripción de dispositivos ortopédicos tales como plantillas, inmovilización con férula de yeso o CAM Walker de 4-6 semanas, o una segunda infiltración o valorar infiltrar otras compuestos como BTX-A ${ }^{10}$.

En cuanto a evidencia científica de los tratamientos más conservadores, encontramos que existe evidencia fuerte de que las plantillas, prefabricadas o a medida, con apoyo del arco medial y las taloneras o modificación del calzado para amortiguación del talón especialmente en aquellos con capacidad de absorción de choque disminuida, producen una reducción del dolor y mejoría en 3 meses $^{3}$. En algunos estudios las plantillas prefabricadas han demostrado la misma efectividad que otras más costosas ${ }^{15}$, mientras que otros concluyen que las personalizadas son las más útiles ${ }^{26}$. Así también, con el uso de férulas nocturnas combinado o no con las anteriores, durante 1-3 meses ${ }^{3}$.

También encontramos una evidencia fuerte en el ejercicio terapéutico dirigido al estiramiento de la fascia plantar y el tríceps sural, y en la terapia manual orientada a las movilizaciones de tobillo pie (dorsiflexión), tejidos blandos fascia plantar y tríceps sural (puntos gatillo y áreas de restricción de movilidad), y el uso de taping con cinta antipronante ${ }^{3}$.

Con una evidencia moderada encontramos el uso de agentes físicos como la iontoforesis, el láser y la fonoforesis en dolor agudo ${ }^{3,27}$. Algunos autores consideran el uso de ortesis plantares, férulas nocturas y el taping con un nivel de evidencia moderada.

El tercer escalón terapéutico, tras 6 meses de evolución del dolor o no mejoría con los tratamientos previos durante 2 ó 3 meses, consiste en valorar tratamiento con ondas de choque o intervención quirúrgica ${ }^{10}$.

El tratamiento con ondas de choque extracorpóreas, se plantea en un tercer escalón en lesiones de más de 6 meses de evolución, tras fracaso de otros tratamientos - como alternativa a métodos más invasivos como infiltraciones o la cirugía. Se recomienda tratar la fascitis plantar con ondas de choque radiales. Las ondas de choque focales y las terapias con ultrasonido pueden considerarse como alternativas de tratamiento ${ }^{29}$.
En cuanto al tratamiento con infiltraciones en fascitis plantar, encontramos en la literatura más de 10 sustancias inyectables entre las que encontramos corticoides, plasma rico en plaquetas (PRP), toxina botulínica-A (BTX-A), membrana amniótica deshidratada, ácido hialurónico, proloterapia... ${ }^{25} \mathrm{Y}$ encontramos mejores resultados con infiltraciones guiadas por ecografía $^{25,30}$.

En la literatura ha demostrado tener evidencia de eficacia a corto plazo la infiltración de corticoide ${ }^{31}$ mientras que la BTX-A y el PRP obtienen buenos resultados para dolencias de más de seis meses de evolución con fallo de tratamiento conservador ${ }^{32,} 33,34$. Además encontramos otras alternativas en estudio como membrana amniótica deshidratada con buenos resultados $^{25}$.

Por último, diversos autores encuentran mejores resultados con la combinación de distintas técnicas, como terapias físicas, tratamientos manuales e infiltraciones, así como la utilidad de las ondas de choque cuando otras terapias fracasan ${ }^{33,35}$. También debemos tener en cuenta que la gran mayoría de las talalgias se resuelven en 1 año sin ningún tipo de intervención y el $90 \%$ de los pacientes mejoran con tratamiento conservador.

\section{Conclusión}

La fascitis plantar es una causa muy común de dolor talar, llegando a afectar hasta al $7 \%$ de la población. La literatura apoya un origen multifactorial de la misma con múltiples factores de riesgo. Su diagnóstico es clínico, pudiéndonos apoyar en pruebas complementarias para realizar diagnóstico diferencial con otras entidades o en casos refractarios. Aquí adquiere gran valor la ecografía que también nos permite realizar tratamientos ecoguiados, obteniendo mejores resultados.

Resulta difícil homogeneizar la información por la ausencia de protocolos y guías clínicas actualizadas. La mayoría de las fascitis plantares se resuelven con tratamiento conservador y, para los casos refractarios, existen múltiples tratamientos y dianas terapéuticas que pueden ayudar a resolverlos. 


\section{Bibliografía}

1. Beson P. Plantar fasciopathy: revisiting the risk factors. Foot Ankle Surg 2014 Sep; 20(3):160-5.

2. Taunton JE, Ryan MB, Clement DB, McKenzie DC, Lloyd-Smith DR, Zumbo BD. A retrospective case-control analysis of 2002 running injuries. Br J Sports Med. 2002; 36:95-101.

3. Martin RL, Davenport TE, ReischI SF, McPoil TG, Matheson JW, Wukich DK, et al. American Physical Therapy Association. Heel pain-plantar fasciitis: revision 2014. J Orthop Sports Phys Ther. 2014; 44:A1 33.

4. Schepsis AA, Leach RE, Gorzyca J. Plantar fasciitis. Etiology, treatment, surgical results, and review of the literature. Clin Orthop 1991: 185-96.

5. $\quad$ Netter. Atlas de anatomía humana. Ed. Elsevier. Ed. $7^{\circ} .2019$.

6. Kirkpatrick J, Yassaie O, Mirjalili SA. The plantar calcaneal spur: a review of anatomy, histology, etiology and key associations. J Anat. 2017 Jun; 230(6):743-51.

7. Schneider HP, Baca JM, Carpenter BB, Dayton PD, Fleischer AE, Sachs BD. American College of Foot and Ankle Surgeons Clinical Consensus Statement: Diagnosis and Treatment of Adult Acquired Infracalcaneal Heel Pain The Journal of Foot \& Ankle Surgery. 2017; 57(2): 370-81.

8. Lemont H, Ammirati K, Usen N. Plantar fasciitis: a degenerative process (fasciosis) without inflammation. J Am Podiatr Med Assoc 2003; 93:234-7.

9. Cobden A, Camurcu Y, Sofu H, Ucpunar H. Evaluation of the association between plantar fascitis and hallux valgus. J Am Podiatr Med Asoc 2019; 110(2)

10. Thomas JL, Christensen JC, Kravitz SR, Mendicino RW, Schuberth JM, Vanore JV, et al. The diagnosis and treatment of heel pain: a clinical practice guideline- revision 2010. J Foot Ankle Surg 2010; 49 (3 Suppl): S1-19.

11. Hansen L, Krogh TP, Ellingsen T, Bolvig L, Fredberg U. Long-Term Prognosis of Plantar Fasciitis: A 5- to 15-Year Follow-up Study of 174 Patients With Ultrasound Examination. Orthop J Sports Med 2018 Mar 6; 6(3):2325967118757983.

12. Irving DB, Cook JL, Menz HB. Factors associated with chronic plantar heel pain: a systematic review. J Sci Med Sport 2006; 9(12):11-22, discussion 23-4

13. Werner RA, Gell N, Hartigan A, Wiggerman N, Keyserling WM. Risk factors for plantar fasciitis among assembly plant workers. PM R 2010; 2:110-6.

14. Priesand SJ, Schmidt BM, Ang L, Wrobel JS, Munson M, Ye W, Pop-Busui R. Plantar fasciitis in patients with type 1 and type 2 diabetes: A contemporary cohort study. J Diabetes Complications. 2019 Oct; 33(10):107399.

15. Lareau C. Plantar and Medial Heel Pain: Diagnosis and Management. J Am Acad Orthop Surg 2014; 22:372-80.

16. Ley JC, Mizel MS, Clifford PD, Temple HT. Value of radiographs in the initial evaluation of nontraumatic adult heel pain. Foot Ankle Int 2006; 27:427-30.

17. Nieto-Parga A, Ruibal-Villanueva MC, Nieto-Baltar B, Fernández-Alonso D, Arias-Gonzales M, Iglesias-Castañón A. Estudio mediante Resonancia Magnética de la patología de las partes blandas del pie. Póster presentado en: SERAM; 2012 May 25-28; Granada, España.

18. Monge-García V, Díaz-Llopis IV, Karaleviciute R, Cholbi-Llobell F, García del Río-Beneyto R, Climent-Barberá JM. Utilidad de la medición ecográfica en el diagnóstico de la fascitis plantar. 57 Congreso SERMEF. Sevilla 2019.

19. Cheng JW, Tsai WC, Yu TY, Huang KY. Reproducibility of sonographic measurement of thickness and echogenicity of the plantar fascia. J Clin Ultrasound 2012; 40(1):14-9.

20. Wu J, Zhang YZ, Gao Y, Luo TY. Assessment the reliability of ultrasonography in the imaging of the plantar fascia: a comparative study. BMC Med Imaging 2019 Aug 7; 19(1):62.

21. Geppert MJ, Mizel MS. Management of heel pain in the inflammatory arthritides. Clin Orthop Relat Res 1998 Apr; 349:93-9.

22. Chanda A y McClain S. Mechanical Modeling of Healthy and Diseased Calcaneal Fat Pad Surrogate. Biomimetics 2019 Mar; 4(1):

23. Vega J, Golanó P, Martínez M, Pérez-Carro L, De Prado M. Neuropatías compresivas de tobillo y pie. Bases anatómicas. Orthotips 2006 Oct-Dic; 2(4): 305-20.

24. Chiari B, McNally E. Nerve Entrapment in Ankle and Foot: Ultrasound Imaging. Semin Musculoskelet Radiol 2018; 22:354-63.

25. Tsikopoulos K, Vasiliadis HS, Mavridis D. Injection therapies for plantar fasciopathy ('plantar fasciitis'): a systematic review and network meta-analysis of 22 randomised controlled trials. Br J Sports Med 2016 Nov; 50(22):1367-75.

26. Xu R, Wang Z, Ma T, Ren Z, Jin H. Effect of 3D Printing Individualized Ankle-Foot Orthosis on Plantar Biomechanics and Pain in Patients with Plantar Fasciitis: A Randomized Controlled Trial. Med Sci Monit 2019; 25:1392-400.

27. Berbrayer D, Fredericson M. Update on evidence-based treatments for plantar fasciopathy. PM R 2014; 6(2):159-69.

28. Díaz López AM, Guzmán Carrasco P. Efectividad de distintas terapias físicas en el tratamiento conservador de la fascitis plantar Revisión sistemática. Rev Esp Salud Pública 2014; 88:157-78.

29. Li X, Zhang L, Gu S, Sun J, Qin Z, Yue J, Zhong Y, Ding N, Gao R. Comparative effectiveness of extracorporeal shock wave, ultrasound, low-level laser therapy, noninvasive interactive neurostimulation, and pulsed radiofrequency treatment for treating plantar fasciitis: A systematic review and network meta-analysis. Medicine (Baltimore) 2018 Oct; 97(43):e12819.

30. Li Z, Xia C, Yu A, Qi B. Ultrasound- versus palpation-guided injection of corticosteroid for plantar fasciitis: a meta-analysis. PLoS One 2014 Mar 21; 9(3):e92671.

31. Whittaker GA, Munteanu SE, Menz HB, Bonanno DR, Gerrard JM, Landorf KB. Corticosteroid injection for plantar heel pain: a systematic review and meta-analysis. BMC Musculoskelet Disord 2019 Aug 17; 20(1):378.

32. Díaz-Llopis IV, Rodríguez-Ruíz CM, Mulet-Perry S, Mondéjar-Gómez FJ, Climent-Barberá JM, Cholbi-Llobel F. Randomized controlled study of the efficacy of the injection of botulinum toxin type A versus corticosteroids in chronic plantar fasciitis: results at one and six months. Clin Rehabil 2012 ; 26(7):594-606.

33. Díaz-Llopis IV, Gómez-Gallego D, Mondéjar-Gómez FJ, López-García A, Climent-Barberá JM, Rodríguez-Ruiz CM. Botulinum toxin type A in chronic plantar fasciitis: clinical effects one year after injection. Clin Rehabil 2013; 27(8):681-5.

34. Shetty SH, Dhond A, Arora M, Deore S. Platelet-Rich Plasma Has Better Long-Term Results Than Corticosteroids or Placebo for Chronic Plantar Fasciitis: Randomized Control Trial. J Foot Ankle Surg 2019 Jan; 58(1):42-6.

35. Celik D, Kuş G, Sırma Sö. Joint Mobilization and Stretching Exercise vs Steroid Injection in the Treatment of Plantar Fasciitis: A Randomized Controlled Study. Foot Ankle Int 2016 Feb; 37(2):150-6. 